\title{
A sensory preconditioning effect after a single flavor-flavor pairing
}

\author{
SAM REVUSKY \\ Memorial University of Newfoundland, St. John's, Newfoundland AIC 5S7, Canada
}

\begin{abstract}
Rats received pairings in which vinegar solution was consumed for $5 \mathrm{sec}$ and then another flavored solution (either salt or sugar) was consumed for 5 sec. Later, the second flavored solution was subjected to aversion conditioning through lithium sickness. The result in a subsequent test was that consumption of the vinegar solution was less than that of appropriate controls. This effect was not a noticeable function of the number of flavor-flavor pairings $(1,2,4,8$, or 16) and was significant after a single flavor-flavor pairing.
\end{abstract}

The sensory preconditioning (SPC) procedure has two phases. In the first phase $\mathrm{S} 1$ is followed by $\mathrm{S} 2$, where $\mathrm{S} 1$ and $\mathrm{S} 2$ are CSs. In the second phase, $\mathrm{S} 2$ is followed by a US. SPC is said to occur if S1 elicits a CR similar to the CR conditioned to $S 2$.

SPC can occur after few S1-S2 pairings. Hoffeld, Kendall, Thompson, and Brogden (1960) found the SPC effect was maximal after four S1-S2 pairings and diminished with additional pairings; they used cats, telereceptive CSs, and a shock US. Using rats in a similar experiment, Prewitt (1967) found a maximal effect after four pairings, but it did not diminish with additional pairings. Lavin (1976) adapted the SPC procedure to the flavor aversion paradigm by training rats to drink saccharin (S1) and coffee (S2) solutions in rapid succession and then pairing the coffee (S2) solution with lithium sickness. SPC was measured by testing the preference for the saccharin solution, which had not been directly paired with sickness. When Lavin (1972) compared the effects of 5, 10, 20, and 40 flavorflavor pairings, he found no significant difference, but the trend of his results suggested that, if there was any effect, SPC might be most pronounced with five pairings.

The present experiment deals with the relative effects of $1,2,4,8$, and 16 flavor-flavor pairings and of the concentration of flavoring in the S1 solution on the magnitude of the SPC effect. The experimental design was 2 by 2 by 5 by 2 factorial. Factor $A$ was whether an SPC procedure or a control procedure was used. Factor B was the flavored solution to be directly paired with toxcosis: For half the rats, it was $20.0 \%$ (w/v) sugar solution; for the other half, it was $2.0 \%(\mathrm{w} / \mathrm{v})$ salt $(\mathrm{NaCl})$ solution. Table 1 outlines the different procedures for these factors during both the SPC phase

This research was supported by Grant A8271 from the National Research Council of Canada. I thank Shannon Coombes for his expert administration of the experimental procedures and Elizabeth Noseworthy and Laura Park for assistance. Richard W. Pohl and Gerard M. Martin were kind enough to critically read the manuscript.
Table 1

Description of Factors A and B of the Experimental Design

\begin{tabular}{ccc}
\hline Level & SPC Phase & Aversion Phase \\
\hline a1 b1 & Vinegar $\rightarrow \mathrm{NaCl}$ & $\mathrm{NaCl} \rightarrow \mathrm{LiCl}$ \\
a2 b1 & Vinegar $\rightarrow$ Sugar & $\mathrm{NaCl} \rightarrow \mathrm{LiCl}$ \\
a1 b2 & Vinegar $\rightarrow$ Sugar & Sugar $\rightarrow \mathrm{LiCl}$ \\
a2 b2 & Vinegar $\rightarrow \mathrm{NaCl}$ & Sugar $\rightarrow \mathrm{LiCl}$ \\
\hline
\end{tabular}

and the conditioning phase. Levels a1 and a2 indicate experimental and control procedures, respectively; Level b1 indicates that $\mathrm{NaCl}$ solution was $\mathrm{S} 2$, and Level b2 indicates that sugar solution was S2. Finally, although it is not indicated in Table 1 , each rat was given equal exposure to both sugar and $\mathrm{NaCl}$ solutions during pretraining. For instance, a rat given vinegar $\rightarrow$ sugar pairings was also given access to $\mathrm{NaCl}$ solution in the absence of vinegar solution, so that it was equally familiar with the $\mathrm{NaCl}$ and sugar flavors. This was meant to insure that the aversion to the $\mathrm{S} 2$ solution would be similar for experimental and control groups; otherwise, aversions would be slightly weaker in the experimental groups because they would be more familiar with the S2 flavor (Klein, Mikulka, \& Hamel, 1976; Lubow, 1973; Revusky \& Bedarf, 1967).

Factors $\mathrm{C}$ and $\mathrm{D}$ of the experimental design are too straightforward to be outlined in Table 1. Factor $\mathrm{C}$ was the number of flavor-flavor pairings: $1,2,4,8$, or 16 . Factor $\mathrm{D}$ was the nature of $\mathrm{S} 1$; in all cases, it was vinegar solution, but for half the rats the concentration was $1.0 \%(\mathrm{v} / \mathrm{v})$, and for the other half, the concentration was $4.0 \%$.

\section{METHOD}

\section{Subjects, Groups, and Materials}

The 200 individually housed male Sprague-Dawley rats ranged in ad-lib weight from 163 to $215 \mathrm{~g}$ on the day prior to the experiment and were factorially divided into 40 groups of 5 each according to the 2 by 2 by 5 by 2 experimental design. There were two replications of this experiment. The first replication included three rats in each group subjected to $1.0 \%$ vinegar solution and two rats in each group subjected to $4.0 \%$ vinegar, and 
vice versa in the second replication. There was no noticeable effect of replications, and they were ignored for statistical purposes.

The rats had free access to dry Purina Chow, but access to fluids was restricted to indicated periods. All experimental procedures were administered in the wire-mesh home cages except injections. Fluids for experimental procedures were presented in glass spouts of the type described by Parker and Revusky (1975).

The S1 solution was either $1.0 \%$ or $4.0 \%(v / v)$ of Heinz cider vinegar. The S2 solution was either $2.0 \%(\mathrm{w} / \mathrm{v})$ of $\mathrm{NaCl}$ (commercial table salt) or $20.0 \%(\mathrm{w} / \mathrm{v})$ of sucrose (commercial sugar). The lithium chloride ( $\mathrm{LiCl}$ ) toxin was administered intraperitoneally in a $2.0 \%(\mathrm{w} / \mathrm{v})$ solution so that the injections contained $20 \mathrm{mg} / \mathrm{ml}$.

\section{Adaptation}

The rats were first deprived of water for 2 days. Then, for another 2 days, they were allowed $10 \mathrm{~min} /$ day of access to unflavored water. If they failed to drink at least $5 \mathrm{~g}$, they were given additional 10-min sessions until they did so. On the next day, each rat was subjected to eight water-water pairings administered at least 30 min apart. First one spout of unflavored water was presented and was removed $5 \mathrm{sec}$ after drinking began. After $2 \mathrm{sec}$, a second spout of unflavored water was presented in the same way for another $5 \mathrm{sec}$ of drinking. These presentations were made by hand and were timed by stopwatch. After a few trials, the rats eagerly waited for the second spout after the first had been removed and began drinking immediately upon its presentation. After the eight water-water pairings were completed, the rats were given $5 \mathrm{~min}$ free access to unflavored water.

\section{Sensory Preconditioning}

Each rat was administered four pairings/day for 4 days. These pairings were either water-water pairings, as in the adaptation phase, or similar pairings in which the vinegar S1 solution was in the first spout and the $\mathrm{NaCl}$ or sugar $\mathrm{S} 2$ solution was in the second spout. The rats designated to receive $16 \mathrm{~S} 1-\mathrm{S} 2$ pairings did not receive any water-water pairings in this phase; the remaining rats received successive water-water pairings until they could receive their designated number of $\mathrm{S} 1-\mathrm{S} 2$ pairings in succession. At least 15 min prior to each S1-S2 pairing, the rats received $5 \mathrm{sec}$ access to the flavored solution that did not have the role of $\mathrm{S} 2$; for instance, rats assigned to vinegar $\rightarrow$ sugar pairings received $5 \mathrm{sec}$ access to $\mathrm{NaCl}$ solution. There was no counterpart to this 5 -sec access period when rats were administered water-water pairings. As in the adaptation phase, the interval between pairings was at least $30 \mathrm{~min}$, and the rats received $5 \mathrm{~min}$ access to unflavored water after the daily pairings were completed.

\section{Flavor Aversion Training}

During each of the 3 days following SPC, the rats were allowed $5 \mathrm{~min}$ to drink the flavored solution designated to be paired with lithium sickness. If $2 \mathrm{~g}$ had not been consumed, $2 \mathrm{ml}$ of the flavored solution were passed over the rat's tongue by means of a syringe as the bottle was removed. Each rat was then injected with $\mathrm{LiCl}$ solution. The dose was $.5 \mathrm{ml}$ of $2.0 \%$ $\mathrm{LiCl}$ solution after the first trial, $1.5 \mathrm{ml}$ after the second trial, and $2.5 \mathrm{ml}$ after the third trial $(10.0,30.0$, and $50.0 \mathrm{mg}$ of $\mathrm{LiCl}$, respectively). About $6.5 \mathrm{~h}$ after the second trial, the rats were given $10 \mathrm{~min}$ free access to unflavored water; 20 of the 200 rats did not drink $5 \mathrm{~g}$ of unflavored water (probably due to adipsia resulting from lithium sickness) and were administered additional sessions until they did so.

The mean amount of $\mathrm{NaCl}$ solution consumed by the rats trained to have an aversion to it was $9.98 \mathrm{~g}$ on the first trial, $2.63 \mathrm{~g}$ on the second trial, and $.67 \mathrm{~g}$ on the third trial. The corresponding mean amounts of sugar solution consumed were $10.56,3.61$, and $.75 \mathrm{~g}$.

\section{Testing}

Beginning 2.0-6.5 h after the final $\mathrm{NaCl}$ or sugar aversion trial, the rats were given 2 days free access to unflavored water and then were completely deprived of fluid for 3 successive days. On each of the next 3 days, they were given $10 \mathrm{~min}$ daily access to unflavored water. Testing on the following day consisted of $10 \mathrm{~min}$ access to the earlier concentration of vinegar solution.

\section{RESULTS AND DISCUSSION}

Figure 1 shows the mean consumption of the vinegar solution on the test day. Each quadrant shows results for SPC and control rats (Factor A of the experimental design) subjected to different numbers of S1-S2 pairings (Factor C). The quadrants are arranged factorially according to the levels of Factors $\mathrm{B}$ (rows) and D (columns). The top row shows results when $\mathrm{NaCl}$ was paired with $\mathrm{LiCl}$, and the bottom row shows results when sugar was paired with $\mathrm{LiCl}$. The left column shows results when $1.0 \%$ vinegar was the $\mathrm{S} 1$ solution, and the right column, when $4.0 \%$ vinegar was S1. Statistical analysis was through four-dimensional ANOVAs performed in each of two ways for the sake of generality: The first way was simply to use the amount consumed by each rat as a score; the second was to use the logarithm of this amount as shown in Table 2. The degrees of freedom for error were 168 throughout and the degrees of freedom for each effect are shown. Each of the main effects shown in Table 2 was significant at $p<.005$ regardless of the type of ANOVA. No interactions were significant in the ANOVA based on

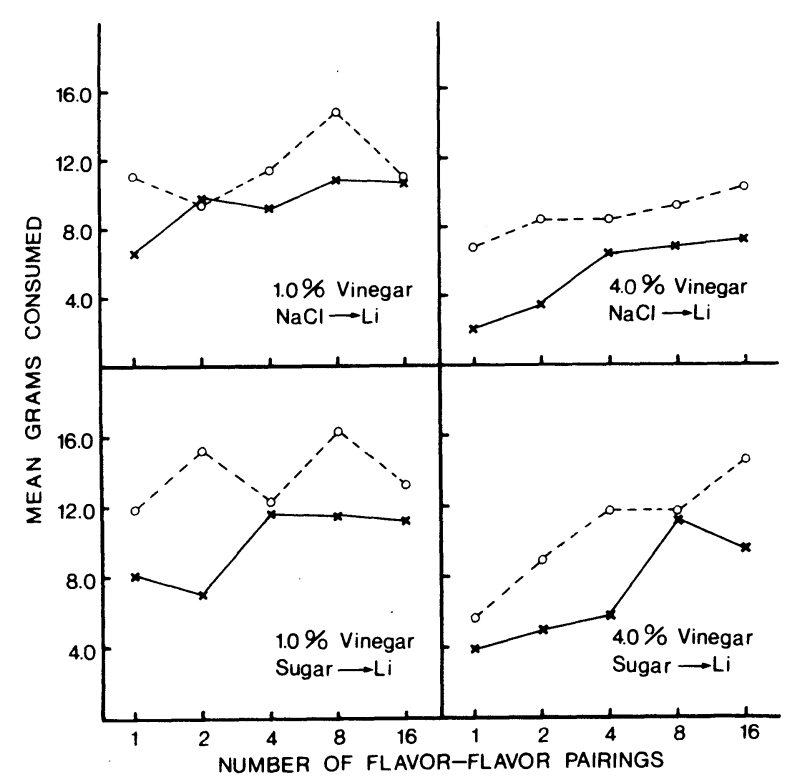

Figure 1. Amount of vinegar solution consumed for rats subjected to an SPC procedure (crosses in solid line) and those subjected to a control procedure (circles in dashed lines). The different quadrants show data for different vinegar concentrations and different $\mathbf{S 2}$ flavors. 
Table 2

Results of ANOVAs for Vinegar-Consumption Tests Based on Absolute Amount Consumed or on its Logarithm

\begin{tabular}{lccc} 
& & \multicolumn{2}{c}{ F Values } \\
\cline { 3 - 4 } \multicolumn{1}{c}{ Factor } & df & Absolute & Log \\
\hline A: SPC vs. Control & 1 & $48.0 \dagger$ & $51.9 \dagger$ \\
B: NaCl $\rightarrow$ LiCl vs. Sugar $\rightarrow$ LiCl & 1 & $15.9 \dagger$ & $10.3^{* *}$ \\
C: Number of S1-S2 Pairings & 4 & $15.8 \dagger$ & $18.8 \dagger$ \\
D: $1 \%$ Vinegar vs. 4\% Vinegar in S1 & 1 & $50.3 \dagger$ & $57.9 \dagger$ \\
A by B & 1 & 1.50 & .04 \\
A by C & 4 & .26 & 1.90 \\
A by D & 1 & .58 & $6.42^{*}$ \\
B by C & 4 & .26 & .19 \\
B by D & 1 & .05 & .79 \\
C by D & 4 & 1.58 & $3.31^{*}$ \\
A by B by C & 4 & .82 & .90 \\
A by B by D & 1 & 1.80 & 2.98 \\
B by C by D & 4 & .81 & .62 \\
A by B by C by D & 4 & 2.07 & 2.01 \\
\hline
\end{tabular}

${ }^{*} p<.025 . \quad{ }^{* *} p<.005 . \quad t p<.001$.

absolute consumption, and only two interactions were significant when logarithms were used; even these interactions were far weaker than the main effects. The significant A effect indicates that the SPC was obtained. The significant main effects of Factors B, C, and D do not indicate that they had any effect on SPC. Their effects on SPC would be manifested through interactions of these factors with Factor A, not by their main effects. The $\mathrm{B}$ effect, lower vinegar consumption if $\mathrm{NaCl}$ was paired with $\mathrm{LiCl}$ than if sugar was paired with $\mathrm{LiCl}$, reflects more stimulus generalization between vinegar and $\mathrm{NaCl}$ than between vinegar and sugar (Parker, 1979). The $\mathrm{C}$ effect, increased vinegar consumption with more S1-S2 pairings, reflects the increase in preference produced by increased familiarity with a flavor (Revusky \& Bedarf, 1967). The D effect was that the rats tended to drink less of the $4.0 \%$ vinegar solution than of the $1.0 \%$ vinegar solution.

The main findings were the lack of any apparent change in the magnitude of the SPC effect as a function of S1-S2 pairings and that one pairing was adequate to produce SPC. The latter was confirmed through a 2 by 2 by 2 ANOVA (Factors A, B, and D of the ANOVA in Table 2) using only the data from rats subjected to a single SPC pairing $[F(1,32)=9.96$ on an absolute basis and $F(1,32)=11.93$ on a logarithmic basis, ps $<.005$ ] Although Hoffeld et al. (1960) claimed to have obtained SPC in cats after a single pairing, they had no specific statistical comparison to prove it.

Minor details are as follows. The significant A by D interaction obtained in the ANOVA on logarithms means that the decrement in vinegar consumption produced by the SPC procedure was proportionately greater when the vinegar concentration was $4.0 \%$ than when it was $1.0 \%$. The significant $\mathrm{C}$ by $\mathrm{D}$ interaction obtained with the ANOVA on logarithms means that the effect of the number of prior S1-S2 pairings on vinegar consumption was proportionately more marked when the concentration of vinegar was $4.0 \%$ than when it was $1.0 \%$.

Finally, I must show the results cannot be artifactual. As Lavin (1972) pointed out, SPC results from two associations: the S1-S2 association and the S2-US association. Increased prior exposure to S2 is likely to weaken the capacity of S2-US pairings to produce the S2-US association (Lubow, 1973). If so, SPC rats, subjected to S1-S2 pairings prior to S2-US pairings, would be bound to develop weaker S2-US associations than controls subjected to S2-US pairings without prior exposure to $\mathrm{S} 2$. Thus presentations of S2 alone were added to the control procedures. For instance, when SPC rats were subjected to vinegar $\rightarrow$ sugar pairings to be followed by sugar $\rightarrow \mathrm{LiCl}$ pairings, the controls were subjected to vinegar $\rightarrow \mathrm{NaCl}$ pairings instead of vinegar $\rightarrow$ sugar pairings. Alone, this control procedure would have been likely to produce stronger sugar aversions among the controls than among the SPC rats because they would have been less familiar with sugar (Klein et al., 1976). To balance this extraneous factor, such controls received equivalent exposure to sugar solution alone prior to the sugar $\rightarrow \mathrm{LiCl}$ pairing. However, it was conceivable that this balancing procedure was excessive, since presentations of S2 alone might produce more latent inhibition than S1-S2 pairings. For instance, with vinegar $\rightarrow$ sugar pairings, the taste of vinegar might conceivably interact with the taste of sugar, or the vinegar might act as an external inhibitor (Pavlov, 1927) and interfere with latent inhibition of the sugar taste. If so, the sugar S2 solution might be more novel to such SPC rats than to their controls and produce stronger aversions to S2, which might, through stimulus generalization, yield stronger aversions to S1 even without any SPC effect. Fortunately, the data were insignificantly contrary to any such hypothesis. On Training Day 2, the mean consumption of the S2 solution among the SPC rats was $3.24 \mathrm{~g}$, and that among the controls was $3.00 \mathrm{~g}$. The rats drank practically nothing on Training Day 3 , so a test for that day would have been meaningless. Incidentally, in the course of this analysis, I confirmed the latent inhibition effect in flavor aversion learning (Revusky \& Bedarf, 1967). Averaged over all other conditions, the mean consumptions on Day 2 of training after 1, 2, 4, 8 , and 16 pairings were, respectively, $2.79,2.61,3.02$, 3.48 , and $3.68 \mathrm{~g}[\mathrm{~F}(1,160)=6.03, \mathrm{p}<.02$, for the trend based on the logarithm of the number of pairings].

\section{REFERENCES}

Hoffeld, D. R., Kendall, S. B., Thompson, R. F., \& Brogden, W. J. Effect of amount of preconditioning training upon the magnitude of sensory preconditioning. Journal of Experimental Psychology, 1960, 54, 198-204. 
Klein, S. B., Mikulka, P. J., \& Hamel, K. Influence of sucrose preexposure on acquisition of a conditioned aversion. Behavioral Biology, 1976, 16, 99-104.

LAVIN, M. J. The establishment of the sensory preconditioning effect using distinct flavors as sensory preconditioning stimuli. Doctoral dissertation, Northern Illinois University, 1972.

LAVIN, M. J. The establishment of flavor-flavor associations using a sensory preconditioning procedure. Learning and Motivation, 1976, 7, 173-183.

Lubow, R. E. Latent inhibition. Psychological Bulletin, 1973, 79, 398-407.

PARKER, L. A. Suppression of consummatory behavior elicited by a lithium conditioned flavor. Doctoral dissertation, Memorial University of Newfoundland, 1979.
Parker, L. A., \& Revusky, S. Failure of Sprague-Dawley rats to transfer taste-aversions by odor-marking the spout. Behavioral Biology, 1975, 15, 383-387.

Pavlov, I. P. [Conditioned reflexes] (G. V. Anrep, Trans.). Oxford: Oxford University Press, 1927.

PrewitT, E. P. Number of preconditioning trials in sensory preconditioning using CER training. Journal of Comparative and Physiological Psychology, 1967, 64, 360-362.

Revusky, S., \& Bedarf, E. W. Association of illness with the prior ingestion of novel foods. Science, 1967, 155, 219-220.

(Received for publication November 15, 1979.) 